Revue

Revue de l'histoire des religions

del'histoire des religions

$4 \mid 2012$

Varia

«Lamentabili sane exitu » (1907). Les documents préparatoires du Saint Office, Claus ARNOLD et Giacomo Losito éditeurs

Rome, Libreria Editrice Vaticana (« Fontes Archivi Sancti Officii

Romani », 6), 2011, 545 p., 24 cm, $60 €$, ISBN 978-88-209-8587-5.

Ward De Pril

OpenEdition

Journals

Édition électronique

URL : http://journals.openedition.org/rhr/8024

DOI : $10.4000 /$ rhr.8024

ISSN : 2105-2573

Éditeur

Armand Colin

Édition imprimée

Date de publication : 1 décembre 2012

Pagination : 566-569

ISBN : 978-2200-92796-7

ISSN : 0035-1423

Référence électronique

Ward De Pril, « «Lamentabili sane exitu » (1907). Les documents préparatoires du Saint Office, Claus ARNold et Giacomo Losito éditeurs », Revue de l'histoire des religions [En ligne], 4 | 2012, mis en ligne le 24 janvier 2013, consulté le 22 septembre 2020. URL : http://journals.openedition.org/rhr/8024 ; DOI : https://doi.org/10.4000/rhr.8024

Ce document a été généré automatiquement le 22 septembre 2020.

Tous droits réservés 


\section{«Lamentabili sane exitu » (1907). Les documents préparatoires du Saint Office, Claus ARNOLD et Giacomo LOSITO éditeurs}

Rome, Libreria Editrice Vaticana (« Fontes Archivi Sancti Officii Romani », 6), 2011, 545 p., 24 cm, $60 €$, ISBN 978-88-209-8587-5.

Ward De Pril

\section{RÉFÉRENCE}

«Lamentabili sane exitu (1907). Les documents préparatoires du Saint Office, Claus ARNOLD etGiacomo LOSITO éditeurs, Rome, Libreria Editrice Vaticana (« Fontes Archivi Sancti Officii Romani », 6), 2011, 545 p., 24 cm, 60 €, ISBN 978-88-209-8587-5.

1 Ce volume, qui traite de la genèse du décret Lamentabili, est la suite logique de l'ouvrage des mêmes auteurs sur la censure d'Alfred Loisy (Libreria Editrice Vaticana, 2009). Celle-ci ne constituait que la moitié de l'intervention prévue par le Saint-Office contre Loisy ; une semaine après la décision d'une mise à l'Index, le Saint-Office confiait aux consulteurs Domenico Palmieri et Pie de Langogne la tâche de rédiger un Elenchus errorum (une liste des erreurs) de Loisy : le début d'une procédure qui allait aboutir au décret Lamentabili. Ce processus est décrit de façon détaillée dans la contribution de Claus Arnold : «Lamentabili sane existu (1907). Le magistère romain et l'exégèse d'Alfred Loisy ".

2 Pour la liste finale de 65 propositions condamnées par le décret Lamentabili, on a puisé principalement dans quatre documents préparatoires, écrits entre avril 1904 et juillet 1907. D’abord, chaque consulteur a composé une liste précédée par une introduction élaborée, de 93 propositions pour Palmieri et 119 pour Pie de Langogne. Les deux documents témoignent clairement d'une tendance intransigeante. Pie de 
Langogne, contrairement à Palmieri, ne prévoyait pas de qualification théologique ; son but était plutôt de rédiger un document de condamnation indifférenciée, à l'exemple du Syllabusde 1864. Au printemps de 1905, ces deux listes étaient fondues - grâce à l'aide de Willem van Rossum - dans un Elenchus unicus, le troisième document crucial, basé principalement sur le texte de Pie de Langogne et très proche déjà, par sa structure, du texte définitif de Lamentabili.

3 À partir de juillet 1905, les propositions de l'Elenchus unicus ont été débattues l'une après l'autre en réunions régulières par la commission des consulteurs du Saint-Office. Les cardinaux de la congrégation ne disposèrent d'une synthèse imprimée de ces travaux que presque une année plus tard (mars 1906), qu'ils examinèrent pour aboutir à un nouvel imprimé transmis aux consulteurs en septembre 1906. Lors des réunions de ces derniers - avant et après l'analyse des cardinaux - ce sont surtout les interventions modératrices d'Alberto Lepidi qui frappent le lecteur: le maitre du Sacré Palais se présente comme une sorte d'antagoniste de Pie de Langogne, tentant de s'opposer au programme des anti-modernistes. Durant le premier semestre 1907, les cardinaux du Saint-Office se saisirent à nouveau du dossier, à l'ordre du jour de leurs réunions hebdomadaires, jusqu'à la rédaction des Propositiones damnandae - quatrième et dernier document décisif dans les préparatifs de Lamentabili -, dont l'élaboration ultime fut confiée au jésuite Steinhuber.

C. Arnold montre que la compréhension de la genèse de Lamentabili, avec sa lenteur, sa complexité et les compromis finaux, est d'une importance capitale pour l'interprétation du décret: il conclut que "l'on n'arriva pas à trouver une solution commune et cohérente du problème du rapport entre l'histoire et le dogme » (p. 32). La genèse difficile et le caractère atténué de Lamentabili sont en fort contraste avec la condamnation intégrale et rapide du modernisme dans l'encyclique Pascendi, née à l'extérieur du Saint-Office. Lamentabili n'est pas un document libéral, «mais en quelque façon, sa genèse porte déjà en elle l'histoire de son dépassement » par l'encyclique.

Comme dans le volume La censure d'Alfred Loisy, l'analyse de la genèse des documents édités par C. Arnold est complétée par une contribution de G. Losito sur les réseaux antimodernistes français dans leur lutte contre Loisy: "Le décret Lamentabili, un document "français". Pie de Langogne entre antimodernistes intransigeants, modérés et novateurs ». Il se concentre particulièrement sur la figure du capucin français Pie de Langogne, qui a joué un rôle crucial dans la réalisation du décret Lamentabili. Plus de la moitié des 65 propositions sont puisées dans l'Elenchus de Pie et il a obtenu que soient visés non seulement les ouvrages de Loisy, mais aussi ceux d'autres neoterici comme $\mathrm{M}^{\mathrm{gr}}$ Mignot, Édouard Le Roy ou Albert Houtin. D'abord, G. Losito montre les liens entre Pie de Langogne et les intransigeants français Alfred Leclerc - supérieur général des Frères de Saint-Vincent-de-Paul - et son confrère Charles Maignen - auteur de plusieurs articles contre Loisy dans La Vérité française, exploités par Pie dans son Elenchus. La correspondance (surtout entre Leclerc et Maignen) nous offre une vue unique sur les démarches, stratégies et conceptions dans les milieux intransigeants. Ensuite, G. Losito aborde l'unicité et l'originalité des positions de Pie de Langogne dans sa lutte contre les neoterici, dénomination par laquelle il vise non seulement Loisy et autres critiques de la Bible, mais aussi - par exemple - des représentants de la démocratie chrétienne (Paul Naudet et Pierre Dabry) ou des défenseurs de Loisy à l'intérieur de l'Église ( $\mathrm{M}^{\mathrm{gr}}$ Mignot et $\mathrm{M}^{\mathrm{gr}}$ Lacroix). Ce faisant, Pie de Langogne a fortement étendu la portée des erreurs stigmatisées ; mais d'autre part, il n'a pas omis de notifier de façon positive les critiques 
«progressistes» de Loisy (notamment Joseph-Marie Lagrange) et de Le Roy (notamment Joannes Wehrlé). Enfin, G. Losito analyse la réception du décret Lamentabili en France, parmi les évêques, dans la presse catholique (revues spécialisées, quotidiens et hebdomadaires), et par quelques figures-clés (Blondel, Lagrange) à l'époque de la crise. Il apparaît alors que le décret a donné lieu à des interprétations très variées : tandis que dans les milieux intransigeants il pouvait être présenté comme une condamnation implicite du modernisme, les cercles plus modérés se jugeaient encouragés par l'absence du mot. L'ambiguïté du décret peut être liée à sa nature de " compromis institutionnel », où tant les positions intransigeantes que des opinions plus modérées trouvaient leur expression. Ici, G. Losito distingue un antimodernisme intransigeant (Billot) - qui n'atteindra son but qu'avec Pascendi - et un antimodernisme modéré (Baudrillart) - pour lequel Lamentabili suffisait comme réponse à l'adresse des «modernistes». Ces derniers avaient d'ailleurs des affinités avec les novateurs (Lagrange, Blondel, Batiffol), en ce qui concerne l'essai d'instaurer un modèle de modernité acceptable.

6 L'édition contient, outre les quatre documents capitaux dans la préparation de Lamentabili commentés ci-dessus (Osservazioni sulle opere di Alfredo Loisy de Palmieri, Elenchus complectens praecipuos hodierni rationalismi theologici errores de Pie de Langogne, Elenchus unicus de Palmieri, Pie de Langogne et Van Rossum, enfin Propositiones damnandae dans la rédaction de Steinhuber), le texte imprimé des débats des consulteurs sur l'Elenchus unicus (Votum Reverendissimum Consultorum. Elenchus Propositionum damnandarum iuxta votum Rev. morum P. Pii, Palmieri, Van Rossum), un appendice (De propositionibus reprobandis Appendix altera) de Pie de Langogne faisant le lien avec la discussion française sur l'herméneutique des dogmes, et le texte imprimé des critiques des consulteurs avec leurs dernières corrections suggérées pour les propositions condamnables selon les cardinaux en 1906 (Animadversiones DD. CC. de propositionibus damnandis). Comme dans leur ouvrage précédent, les éditeurs témoignent d'une grande rigueur scientifique. Leurs notes offrent des informations biographiques et bibliographiques, signalent des erreurs ou des fautes dans les documents, évaluent notamment la reproduction, la traduction ou l'interprétation des textes sources de Loisy dans les Elenchi. L'ensemble apporte beaucoup à la compréhension et à l'interprétation du dossier. S'ajoutent une synopse des propositions condamnées par le décret Lamentabili et de celles contenues dans les listes préparatoires, ainsi qu'une liste des propositions contenues dans l'Elenchus unicus mais écartées du texte définitif de Lamentabili. Le volume, qui s'ouvre par un avant-propos d'Émile Poulat, constitue donc un recueil très étoffé, qui met à la disposition des spécialistes de la crise moderniste des documents cruciaux pour la préparation du décret Lamentabili (surtout l'Elenchus de Pie de Langogne), accompagnés d'un aperçu de son histoire (genèse, liens avec l'antimodernisme français) et d'hypothèses pour son interprétation. 


\section{AUTEURS}

WARD DE PRIL

Université de Louvain. 